\title{
Room temperature THz detection and emission with semiconductor nanodevices
}

DOI:

10.1109/CDE.2013.6481381

Link to publication record in Manchester Research Explorer

\section{Citation for published version (APA):}

Mateos, J., Millithaler, J. F., Iniguez-de-la-Torre, I., Iniguez-de-la-Torre, A., Vasallo, B. G., Perez, S., Gonzalez, T., Alimi, Y., Zhang, L., Rezazadeh, A., Song, A. M., Sangare, P., Ducournau, G., Gaquiere, C., Westlund, A., \& Grahn, J. (2013). Room temperature THz detection and emission with semiconductor nanodevices. In host publication (pp. 215-218). https://doi.org/10.1109/CDE.2013.6481381

\section{Published in:}

host publication

\section{Citing this paper}

Please note that where the full-text provided on Manchester Research Explorer is the Author Accepted Manuscript or Proof version this may differ from the final Published version. If citing, it is advised that you check and use the publisher's definitive version.

\section{General rights}

Copyright and moral rights for the publications made accessible in the Research Explorer are retained by the authors and/or other copyright owners and it is a condition of accessing publications that users recognise and abide by the legal requirements associated with these rights.

\section{Takedown policy}

If you believe that this document breaches copyright please refer to the University of Manchester's Takedown Procedures [http://man.ac.uk/04Y6Bo] or contact uml.scholarlycommunications@manchester.ac.uk providing relevant details, so we can investigate your claim.

\section{OPEN ACCESS}




\section{Room Temperature $\mathrm{THz}$ Detection and Emission with Semiconductor Nanodevices}

\author{
J. Mateos, J. F. Millithaler, I. Íñiguez-de-la-Torre, \\ A. Íñiguez-de-la-Torre, B. G. Vasallo, S. Pérez, \\ T. González \\ Departamento de Física Aplicada \\ Universidad de Salamanca \\ Salamanca, Spain
}

\author{
P. Sangare, G. Ducournau, C. Gaquiére \\ Institut d'Electronique, Microélectronique et de \\ Nanotechnologies (IEMN) \\ Villeneuve D'Ascq, France
}

\author{
Y. Alimi, L. Zhang, A. Rezazadeh, A. M. Song \\ School of Electrical \& Electronic Engineering \\ The University of Manchester \\ Manchester, UK
}

A. Westlund, J. Grahn

Microtechnology and Nanoscience - MC2

Chalmers University of Technology

Gothenburg, Sweden

\begin{abstract}
In this paper we present the advances on the fabrication of $\mathrm{THz}$ emitters and detectors obtained within the framework of the European ROOTHz project. Two types of devices are explored, self-switching diodes and slot-diodes, using both narrow bandgap and wide bandgap semiconductors. This broad approach allows us to improve the frequency and power generated by Gunn diodes and the responsivity and noise of detectors at $\mathrm{THz}$ frequencies.
\end{abstract}

Keywords-THz devices; Gunn oscillations; nanodevices; Monte Carlo simulation

\section{INTRODUCTION}

The field of Terahertz Science and Technology is gaining international interest due to its numerous applications ranging from ultra high speed optical transmission systems to medical diagnosys, industrial quality control and security-screening tools. In this field, the efforts of electronics industry are centered on device scaling down to the nanometer range to increase the operational speed. In spite of the enormous technological efforts, the "THz gap" is still not covered, since the only reduction of the gate length is not enough to increase the cut-off frequency as predicted by the traditional scaling rules. On the other hand, since the quanta of terahertz radiation are much smaller than the thermal energy at room temperature (RT), photonic devices based on interband or intersubband transitions are operational only at cryogenic temperature.

The $\mathrm{THz}$ range is an intermediate range of wavelengths that will open a new area of systems directly related to information and communication technologies, significantly extending the present ones based on photonic and electronic devices. Thus solid-state $\mathrm{THz}$ devices can be either considered as belonging to both fields or to none of them. Indeed the wide application area of $\mathrm{THz}$ for astronomy, environment, communications,

This work has been partially supported by European Commission through the ROOTHz Project ICT-2009-243845, the Dirección General de Investigación (MICINN) through Project TEC2010-15413 and by the Consejería de Educación, Junta de Castilla y León through Project SA183A12-1. imaging, security, biology and medicine could lead to define the $\mathrm{THz}$ range as a specific scientific, engineering and application field [1], [2]. Molecular spectroscopy is very important for astronomy (analysis of the interstellar gas, planetary observations), environment (pollution monitoring), etc. For telecommunications, secure local communications (through high attenuation outside the targeted area) with ultrahigh data rates will be possible. Imaging is an important application for security (weapon and illicit material detection), biology and medicine. The emergence of novel functional $\mathrm{THz}$ devices will be of immense interest for all those applications.

The bottleneck of Terahertz Science and Technology is the fabrication of room temperature, continuous wave, compact, tunable and powerful sources (at low cost, if possible). For this sake we propose to exploit $\mathrm{THz}$ Gunn oscillations in novel (narrow and wide bandgap) semiconductor nanodevices, which have been predicted by simulations but not experimentally confirmed yet. Interestingly, $\mathrm{THz}$ detectors can be fabricated with the same technology, thus allowing designing a simple $\mathrm{THz}$ detection/emission system.

\section{THE DEVICES}

$\mathrm{THz}$ sources can be obtained by the use of frequency multipliers of fundamental oscillators such as RTDs, IMPATT and Gunn diodes. However, the oscillation frequency of classical Gunn diodes (based on the transfer of electrons to the upper valleys) can only be extended to a certain limit, around $300 \mathrm{GHz}$ (associated with the scattering and energy relaxation frequencies). In order to fabricate continuous wave $\mathrm{THz}$ emitters based on Gunn oscillations overcoming this limit, in the framework of the EU funded project $\mathrm{ROOTHz}$ we investigate two new types of nanodevices:

- Slot diodes: Monte Carlo simulations [3] have shown the possibility of generating ultrafast (in the $\mathrm{THz}$ range) Gunn-like oscillations in InGaAs/InAlAs slotdiodes. The simplified geometry of these devices (also called ungated HEMTs) is shown in Fig. 1(b). 
Simulations predict that when the bias applied to the slot diode surpasses a threshold voltage associated to the $\Gamma$-L intervalley energy, the current exhibits an oscillatory behavior at extremely high frequency (much higher than that obtained by the "classical" Gunn effect). Such high frequencies are reached due to the presence of a recess, which concentrates the electric field and injects very fast electrons into the drain region, providing a high field domain that moves towards the drain at the $\Gamma$ electron velocity.

- Self-switching diodes (SSDs): This device was originally proposed by A. Song [4]. By breaking the symmetry of a narrow channel [see Fig. 1(a)] this device provides a rectifying behavior (based on surface and electrostatic effects) without the use of any doping junction or barrier structure and can be fabricated with a simple single-step lithographic process. By downscaling the device dimensions and using highmobility material systems (like those involving channels made of narrow bandgap semiconductors), the operation of SSDs can approach the $\mathrm{THz}$ range at room temperature [5], as experimentally demonstrated in [6] for an array of these devices working as detector up to $110 \mathrm{GHz}$, and more recently beyond $1 \mathrm{THz}$ at room temperature [7].

Interestingly, the special geometry of SSDs also benefits the onset of Gunn oscillations. This will be of great help for the design of GaN-based Gunn diodes, which will provide higher power than traditional GaAs Gunn diodes at very high frequency due to its high saturation velocity, high breakdown field and the possibility of high temperature operation. Indeed, in recent years much effort has been made in the fabrication of "classical" (vertical) GaN Gunn diodes [8], although without success as concerns oscillations, even if simulations predicted their presence. The planar topology of SSDs is expected to solve the problems involving heat dissipation, since the channels can be separated from each in order to limit the power density. The planar geometry also provides important ad-
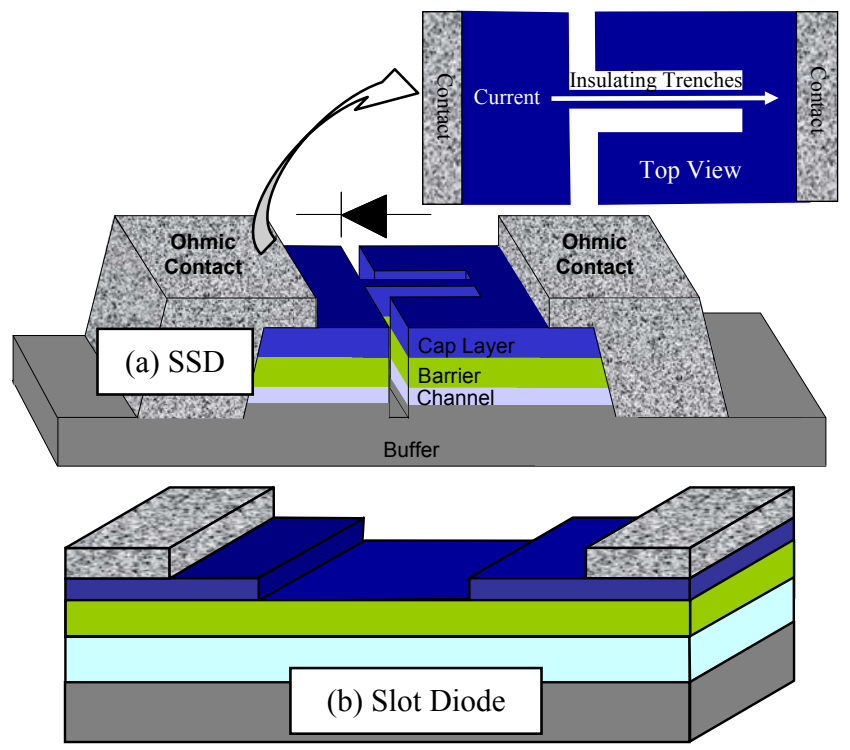

Figure 1. Geometry of (a) self switching diodes (SSDs) and (b) slot diodes vantages over traditional diodes (Schottky barrier or Gunn) used for today's THz systems. In particular, the integration of antennas is very simple, so that a more efficient electromagnetic coupling with free space can be achieved, thus providing higher emitted power and improved detection responsivity.

\section{TECHNOLOGY}

The simplicity of the technological process used for the fabrication of both types of diodes is remarkable, since it only involves the etching of insulating trenches or recess lines on a semiconductor surface (a single step of high resolution lithography). The accurate fabrication of SSDs on the different material systems (examples are given in Figs. 2 and 3) have been possible due to the efforts of the partners of the ROOTHz consortium. It is remarkable that new technologies have been developed within this project, and, for the first time, SSDs based on GaN (IEMN, Fig. 3) and InAs (even if the process is still being improved at Chalmers University) were fabricated.

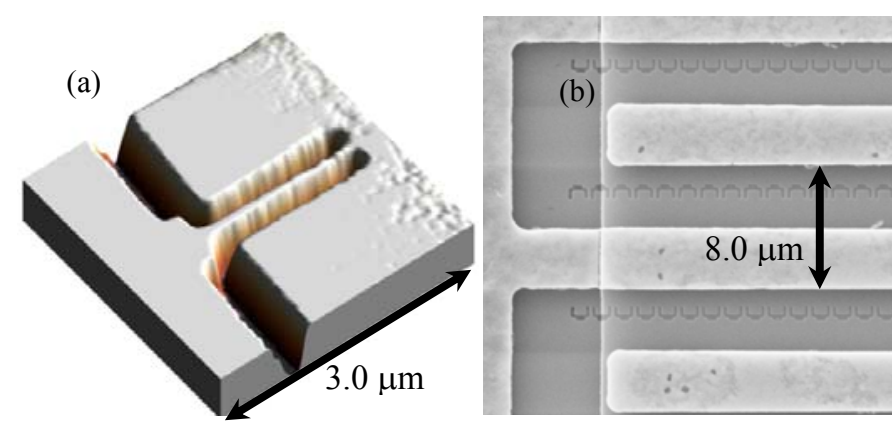

Figure 2. (a) AFM image of a single GaAs SSD [7] and (b) SEM picture of part an array of 2000 InGaAs SSDs, with interdigital contacts [9], both fabricated at the University of Manchester, UK.
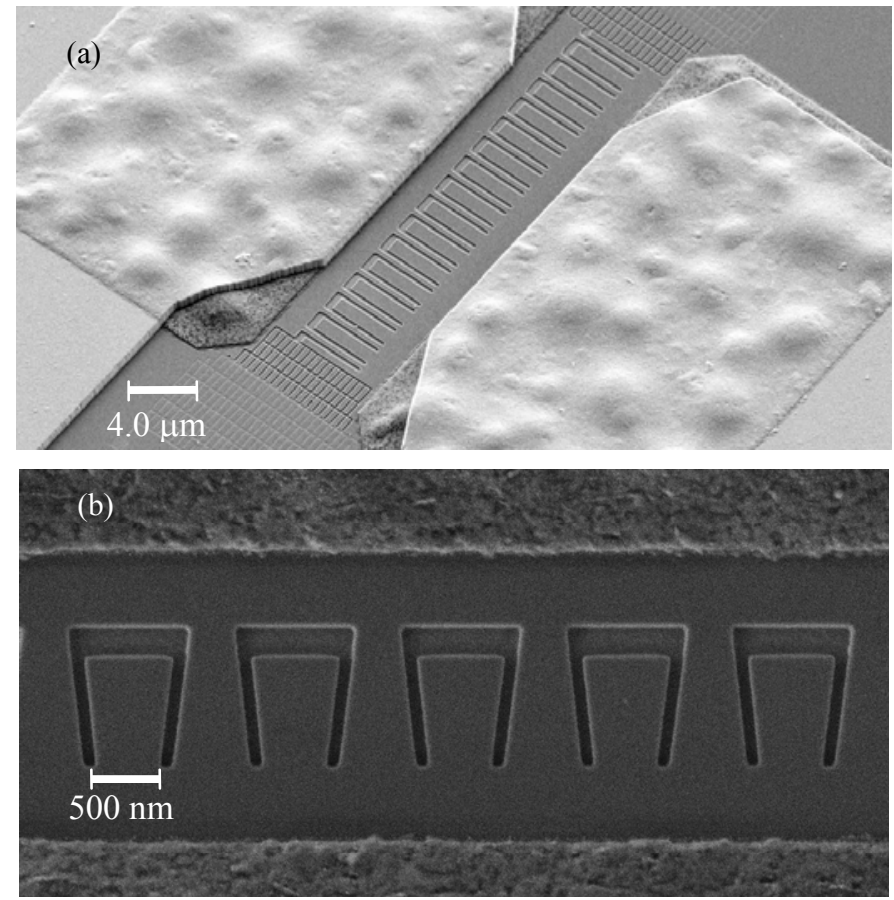

Figure 3. SEM pictures of (a) an array of rectangular SSDs and (b) the newly designed $\mathrm{V}$-shape diodes, both fabricated on GaN at the IEMN, France. 


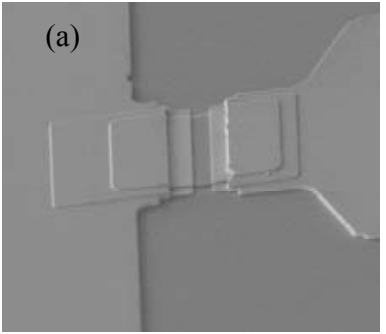

(b)

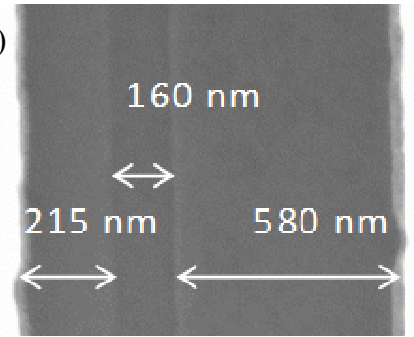

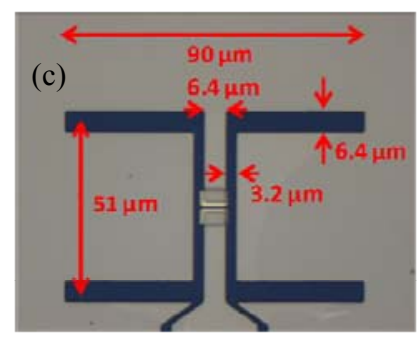

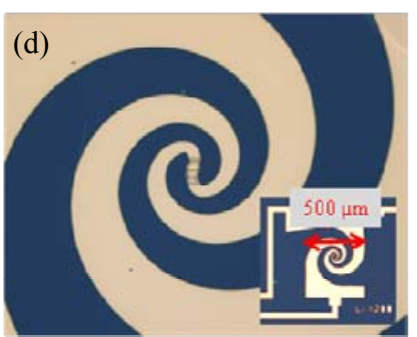

Figure 4. (a) and (b) SEM images of an InGaAs slot diode fabricated at the Chalmers University of Technology, Sweden. Two types of THz antennae have been integrated with the devices, (c) double slot and (d) spiral.

Even if the fabrication of InGaAs slot-diodes is simpler (since it takes advantage of the experience on the same process for the gate recess in HEMTs), we have managed to fabricate devices with different cap layer thicknesses (key parameter for the appearance of ultra-fast Gunn oscillations) and source, recess and drain lengths, Fig. 4. Different types of $\mathrm{THz}$

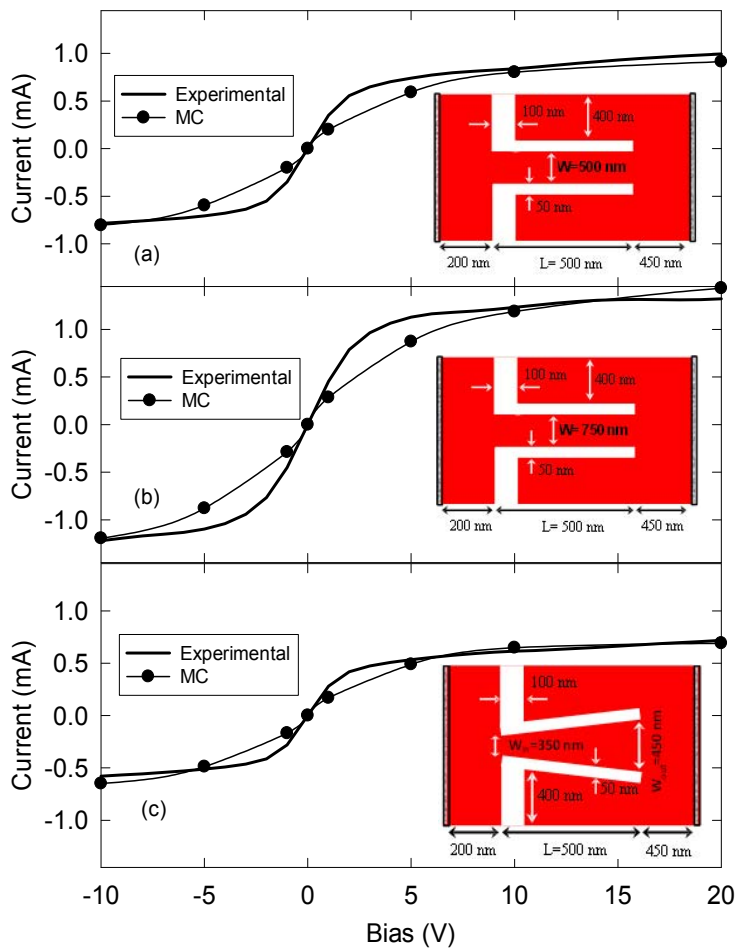

Figure 5. Comparison between experimental and MC simulated I-V curves of arrays of $16 \mathrm{GaN}$ SSDs in parallel (a) and (b) with rectangular geometry and (c) with V-shape (dimensions shown in the insets). antennae (mainly broadband) have been integrated with both SSDs and slot-diodes in order to improve the free-space coupling of the radiation emitted or detected by the devices.

More details on the technological processes are given in [7], [9]-[11].

\section{THz EMITTERS}

Monte Carlo (MC) simulations have been extensively used for the optimization of the design of the devices once the parameters of the models have been adjusted [12]. The good agreement between the measured and simulated I-V curves of GaN SSDs with several geometries is shown in Fig. 5 and for InGaAs slot-diodes in Fig. 6.

Regarding the possibility of generating Gunn oscillations with GaN SSDs (Fig. 7), MC simulations showed that V-shape is the most favorable geometry for the onset of the oscillations, since they appear at low current levels. Indeed, the V-shape diode already exhibits oscillations for an applied voltage of

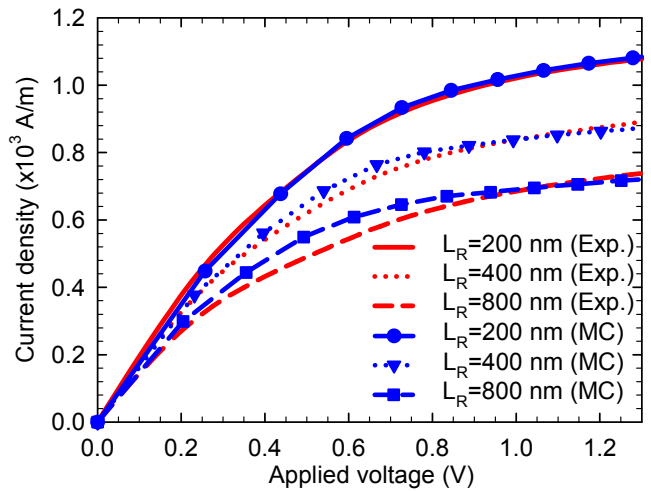

Figure 6. Comparison between the measured and simulated I-V curves of slotdiodes with different recess lenghts.
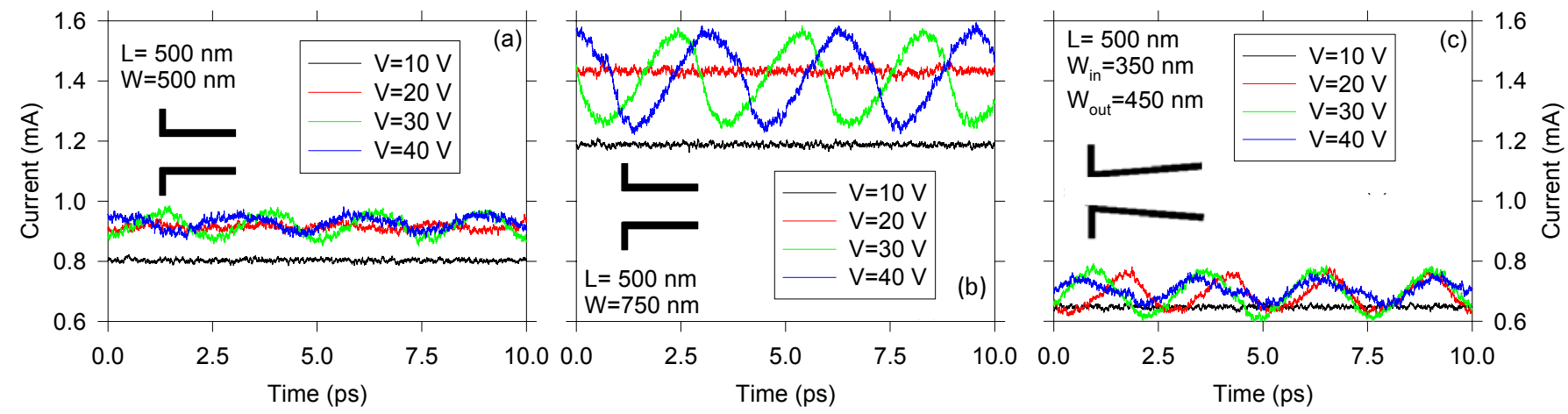

Figure 7. Simulated time-sequences of current in the GaN SSDs with the geometries of Fig. 5 for applied voltages from 10 to $40 \mathrm{~V}$. 
$20 \mathrm{~V}$, while at least $30 \mathrm{~V}$ are necessary in the case of the square diodes. The increase in the width from 500 to $750 \mathrm{~nm}$ significantly enhances the amplitude of the oscillations in the square diode, even if the consumed DC power is higher. Thus, the DC power necessary for the onset of oscillations is of about $14 \mathrm{~mW}$ in the V-shape diodes, as compared with the 27 and $42 \mathrm{~mW}$ in the square diodes of 500 and $750 \mathrm{~nm}$ of width, respectively. This means that less DC power will be dissipated by the V-shape devices and higher efficiencies can be achieved (the amplitude of the oscillation is similar to that of the $500 \mathrm{~nm}$ wide square SSD).

In spite of the expectations generated by the results of MC simulations, all the efforts carried out for the $\mathrm{THz}$ characterization of the fabricated SSDs and slot diodes with diverse geometries (integrated both with coplanar wave accesses and $\mathrm{THz}$ antennae) have not provided any evidence of current oscillations. The reason for this could be that no resonant circuit is attached to the devices, since so far the DC voltage has been directly applied to the terminals of the diodes.

\section{THz DETECTORS}

As any other non-linear device, SSDs can be used as a signal detector. One of their main advantages is that combining the use of high mobility semiconductors (such as InGaAs or InAs) and the parallelization of many SSDs, they can respond up to very high frequencies with a reduced noise level, thus
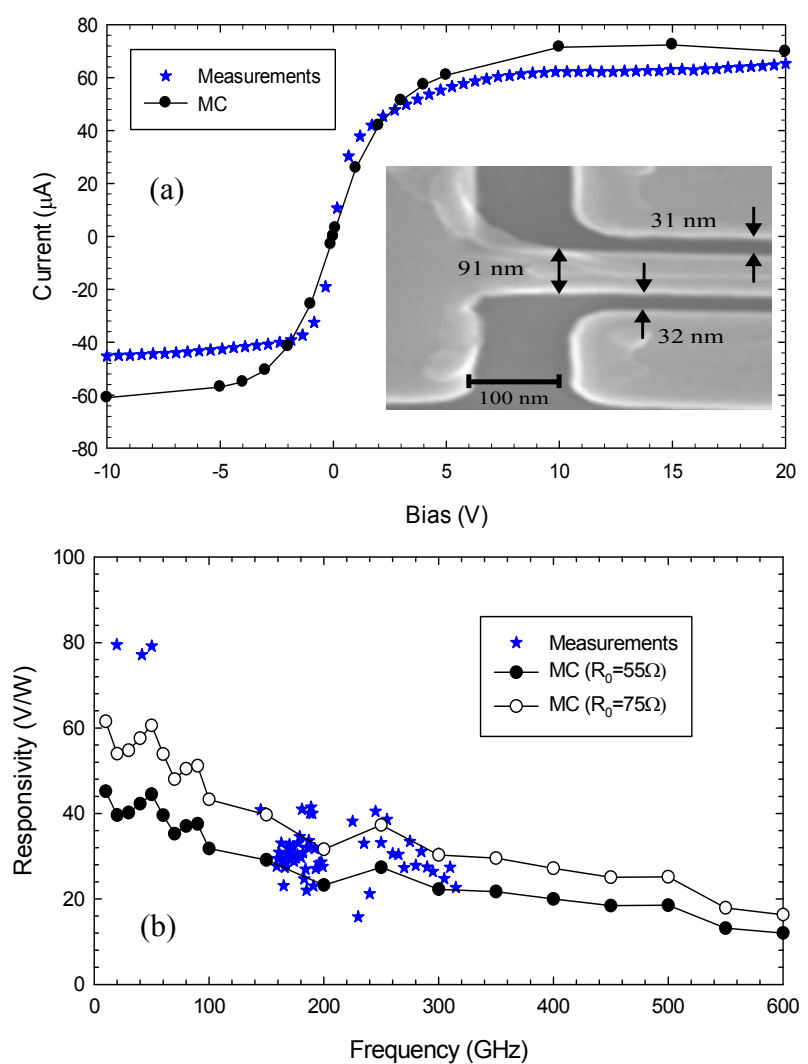

Figure 8. Comparison between experimental results (stars) and Monte Carlo simulations (circles) of the (a) I-V curve and (b) responsivityof one single GaN SSD with geometry optimized for an improved detection $(L=1.0 \mu \mathrm{m}$ and $W=90 \mathrm{~nm})$. Two characteristic line impedances are considered in the $\mathrm{MC}$ simulations $R_{0}=55 \Omega$ (about the experimental value) and $75 \Omega$. Figure taken from [11]. enhancing their sensitivity for detecting $\mathrm{THz}$ signals. In [9] we showed how an array of 2000 InGaAs SSDs, Fig. 2(b), provides a noise equivalent power as low as $65 \mathrm{pW} / \mathrm{Hz}^{1 / 2}$ with a responsivity of about $160 \mathrm{~V} / \mathrm{W} @ 110 \mathrm{GHz}$ at room temperature. These values are not exceptional for such frequency range (which was the limit of the characterization system), their importance lies in the fact that they are expected to remain similar up to the $\mathrm{THz}$ range, thus being at the stateof-the-art for room temperature $\mathrm{THz}$ detection.

Even if $\mathrm{GaN}$ is not the optimum material to be used in SSDs for high frequency detection, GaN SSDs have been fabricated and characterized as detectors as a first step towards the development of a complete room-temperature integrated $\mathrm{THz}$ emitter/detector system. Fig. 8 shows the I-V curve of a GaN SSDs with a narrow channel (in order to increase the nonlinearity and thus the sensitivity for detection) and the responsivity measured under probes for devices with coplanar access lines up to $325 \mathrm{GHz}$. The agreement of the experimental results with MC simulations is very satisfactory and indicates that GaN SSDs can be used as high frequency detectors up to $600 \mathrm{GHz}$, with still much room for optimization. For example, high impedance access lines can increase the extrinsic responsivity, Fig. 8(b), since the matching with the highly resistive SSDs would be much better. In [10] and [11] we have shown how an improvement can also be obtained by using ion implantation for delineating the isolation of the channels (instead of etching sharp trenches, not easy to do in $\mathrm{GaN}$ ).

\section{REFERENCES}

[1] D. Mittleman, Editor, Sensing with THz Radiation, Springer-Verlag, Berlin, 2003.

[2] R. Appleby and H. B. Wallace, "Standoff detection of weapons and contraband in the $100 \mathrm{GHz}$ to $1 \mathrm{THz}$ region," IEEE Trans. Antennas and Propagation, vol. 55, pp. 2944-2956, 2007.

[3] S. Pérez, T. González, D. Pardo and J. Mateos, "Terahertz gunn-like oscillations in InGaAs/InAlAs planar diodes,” J. App. Phys., vol. 103, 094516, 2008.

[4] A. M. Song, M. Missous, P. Omling, A. R. Peaker, L. Samuelson and W. Seifert, "Unidirectional electron flow in a nanometer-scale semiconductor channel: A self-switching device," App. Phys. Lett., vol. 83, pp. 1881-1883, 2003.

[5] J. Mateos, B. G. Vasallo, D. Pardo and T. González, "Operation and high-frequency performance of nanoscale unipolar rectifying diodes," App. Phys. Lett., vol. 86, 212103, 2005.

[6] C. Balocco et al., "Microwave detection at $110 \mathrm{GHz}$ by nanowires with broken symmetry," Nano Letters, vol. 5, pp. 1423-1427, 2005.

[7] C. Balocco et al., "Room-temperature operation of a unipolar nanodiode at terahertz frequencies," App. Phys. Lett., vol. 98, 223501, 2011.

[8] O. Yilmazoglu, K. Mutamba, D. Pavlidis, and T. Karaduman, "First observation of bias oscillations in GaN Gunn diodes on GaN substrate," IEEE Trans. Elec. Dev., vol. 55, pp. 1563-1567, 2008.

[9] C. Balocco, S. R. Kasjoo, L. Q. Zhang, Y. Alimi and A. M. Song, "Lowfrequency noise of unipolar nanorectifiers," App. Phys. Lett., vol. 99, 113511, 2011.

[10] P. Sangaré et al., "GaN-based nano rectifiers for $\mathrm{THz}$ detection," Proceedings of the 37th International Conference on Infrared, Millimeter, and Terahertz Waves (IRMMW-THz), 2012

[11] P. Sangaré et al., "Experimental Demonstration of Direct Terahertz Detection at Room-Temperature in $\mathrm{AlGaN} / \mathrm{GaN}$ Asymmetric Nanochannels", J. App. Phys., in press, DOI: 10.1063/1.4775406, 2013.

[12] A. Iñiguez-de-la-Torre et al., "Searching for THz Gunn oscillations in GaN planar nanodiodes,” J. Appl. Phys., vol. 111, 113705, 2012. 